\title{
Conjugacy of a Discrete Semidynamical System in a Neighbourhood of the Nontrivial Invariant Manifold
}

\author{
Andrejs Reinfelds ${ }^{1,2}$ \\ ${ }^{1}$ Institute of Mathematics and Computer Science, University of Latvia, Raiņa bulvāris 29, Rīga 1459, Latvia \\ ${ }^{2}$ Faculty of Physics and Mathematics, University of Latvia, Zellı iela 8, Rìga 1002, Latvia
}

Correspondence should be addressed to Andrejs Reinfelds; reinf@latnet.lv

Received 21 November 2013; Accepted 15 January 2014; Published 25 February 2014

Academic Editor: Tuncay Candan

Copyright (c) 2014 Andrejs Reinfelds. This is an open access article distributed under the Creative Commons Attribution License, which permits unrestricted use, distribution, and reproduction in any medium, provided the original work is properly cited.

The conjugacy of a discrete semidynamical system and its partially decoupled discrete semidynamical system in a Banach space is proved in a neighbourhood of the nontrivial invariant manifold.

\section{Introduction}

The conjugacy for noninvertible mappings in a Banach space was considered by Aulbach and Garay [1-3]. For noninvertible mappings in a complete metric space it was extended and generalized by Reinfelds [4-9]. In the present paper we consider the case when the linear part of the noninvertible mapping depends on the behaviour of variables in a neighbourhood of the nontrivial invariant manifold.

\section{Invariant Manifold}

Let $\mathbf{E}$ and $\mathbf{F}$ be Banach spaces, $\mathbf{B}(a)=\{r \in \mathbf{F}|| r \mid \leq a\}$, and $a>0$. Consider the following mapping $S: \mathbf{E} \times \mathbf{B}(a) \rightarrow$ $\mathbf{E} \times \mathbf{B}(a)$ defined by

$$
\begin{gathered}
x_{1}=g(x)+\Psi(x, r)=X(x, r), \\
r_{1}=A(x) r+\Phi(x, r)=R(x, r),
\end{gathered}
$$

where the derivative of the diffeomorphism $g: \mathbf{E} \rightarrow \mathbf{E}$ is uniformly continuous $\left\|D g(x)-D g\left(x^{\prime}\right)\right\| \leq \omega\left(\left|x-x^{\prime}\right|\right)$, mappings $A, \Psi$, and $\Phi$ are Lipschitzian,

$$
\begin{gathered}
\left\|A(x)-A\left(x^{\prime}\right)\right\| \leq \gamma\left|x-x^{\prime}\right|, \\
\left|\Psi(x, r)-\Psi\left(x^{\prime}, r^{\prime}\right)\right| \leq \varepsilon\left(\left|x-x^{\prime}\right|+\left|r-r^{\prime}\right|\right), \\
\left|\Phi(x, r)-\Phi\left(x^{\prime}, r^{\prime}\right)\right| \leq \varepsilon\left(\left|x-x^{\prime}\right|+\left|r-r^{\prime}\right|\right), \\
\sup _{x}\|A(x)\|+2 \varepsilon<1, \\
\sup _{x}|\Phi(x, 0)| \leq a\left(1-\sup _{x}\|A(x)\|-\varepsilon\right) .
\end{gathered}
$$

At the beginning we will modify the previous results on the existence of invariant manifolds of Nelmark and Sacker $[10,11]$ for $(1)$.

Lemma 1. If

$$
\begin{aligned}
& \left(\sup _{x}\|A(x)\|+4 \varepsilon+\gamma \frac{\sup _{x}|\Phi(x, 0)|}{1-\sup _{x}\|A(x)\|-\varepsilon}\right) \\
& \quad \times \sup _{x}\left\|D g(x)^{-1}\right\| \leq 1
\end{aligned}
$$

then there exists a continuous mapping $u: \mathbf{E} \rightarrow \mathbf{F}$ satisfying the following properties:

(i) $u(g(x)+\Psi(x, u(x)))=A(x) u(x)+\Phi(x, u(x))$;

(ii) $\left|u(x)-u\left(x^{\prime}\right)\right| \leq\left|x-x^{\prime}\right|$;

(iii) $\|u\| \leq \sup _{x}|\Phi(x, 0)| /\left(1-\sup _{x}\|A(x)\|-\varepsilon\right)$. 
Proof. The set of continuous mappings $u: \mathrm{E} \rightarrow \mathrm{F}$,

$$
\mathscr{K}=\left\{u \in \mathbf{C}(\mathbf{E}, \mathbf{F})\left|\sup _{x}\right| u(x) \mid<+\infty\right\}
$$

equipped with the norm

$$
\|u\|=\sup _{x}|u(x)|
$$

is a Banach space. The set

$$
\mathscr{K}_{1}=\left\{u \in \mathscr{K}|\|u\| \leq a,| u(x)-u\left(x^{\prime}\right)|\leq| x-x^{\prime} \mid\right\}
$$

is a closed subset of the Banach space $\mathscr{K}$.

Let us consider the mapping $u \mapsto \mathscr{L} u, u \in \mathscr{K}_{1}$ defined by the equality

$$
\mathscr{L} u\left(x_{1}\right)=A(x) u(x)+\Phi(x, u(x))
$$

where

$$
x_{1}=g(x)+\Psi(x, u(x))
$$

If $u \in \mathscr{K}_{1}$ then

$$
\|\mathscr{L} u\| \leq\left(\sup _{x}\|A(x)\|+\varepsilon\right)\|u\|+\sup _{x}|\Phi(x, 0)| \leq a .
$$

We have

$$
\begin{aligned}
& \left|\mathscr{L} u\left(x_{1}\right)-\mathscr{L} u^{\prime}\left(x_{1}^{\prime}\right)\right| \\
& \leq \quad\left(\sup _{x}\|A(x)\|+\varepsilon\right)\left\|u-u^{\prime}\right\| \\
& \quad+\left(\sup _{x}\|A(x)\|+2 \varepsilon+\gamma\|u\|\right)\left|x-x^{\prime}\right|, \\
& \left|x-x^{\prime}\right| \\
& \leq \sup _{x}\left\|D g(x)^{-1}\right\|\left|g(x)-g\left(x^{\prime}\right)\right| \\
& =\sup _{x}\left\|D g(x)^{-1}\right\|\left|x_{1}-x_{1}^{\prime}-\Psi(x, u(x))+\Psi\left(x^{\prime}, u^{\prime}\left(x^{\prime}\right)\right)\right| \\
& \leq \sup _{x}\left\|D g(x)^{-1}\right\|\left(\left|x_{1}-x_{1}^{\prime}\right|+2 \varepsilon\left|x-x^{\prime}\right|+\varepsilon\left\|u-u^{\prime}\right\|\right) .
\end{aligned}
$$

It follows

$$
\begin{aligned}
\left|x-x^{\prime}\right| \leq & \frac{\sup _{x}\left\|D g(x)^{-1}\right\|}{1-2 \varepsilon \sup _{x}\left\|D g(x)^{-1}\right\|}\left|x_{1}-x_{1}^{\prime}\right| \\
& +\frac{\varepsilon \sup _{x}\left\|D g(x)^{-1}\right\|}{1-2 \varepsilon \sup _{x}\left\|D g(x)^{-1}\right\|}\left\|u-u^{\prime}\right\| .
\end{aligned}
$$

Then

$$
\begin{aligned}
& \left|\mathscr{L} u\left(x_{1}\right)-\mathscr{L} u^{\prime}\left(x_{1}^{\prime}\right)\right| \\
& \leq\left(\sup _{x}\|A(x)\|+\varepsilon\right. \\
& \left.\quad+\frac{\left(\sup _{x}\|A(x)\|+2 \varepsilon+\gamma\|u\|\right) \sup _{x}\left\|D g(x)^{-1}\right\| \varepsilon}{1-2 \varepsilon \sup _{x}\left\|D g(x)^{-1}\right\|}\right) \\
& \quad \times\left\|u-u^{\prime}\right\| \\
& \quad+\frac{\left(\sup _{x}\|A(x)\|+2 \varepsilon+\gamma\|u\|\right) \sup _{x}\left\|D g(x)^{-1}\right\|}{1-2 \varepsilon \sup _{x}\left\|D g(x)^{-1}\right\|} \\
& \quad \times\left|x_{1}-x_{1}^{\prime}\right| \cdot
\end{aligned}
$$

Let us note that

$$
\frac{\left(\sup _{x}\|A(x)\|+2 \varepsilon+\gamma\|u\|\right) \sup _{x}\left\|D g(x)^{-1}\right\|}{1-2 \varepsilon \sup _{x}\left\|D g(x)^{-1}\right\|} \leq 1 .
$$

We obtain

$$
\begin{aligned}
\left|\mathscr{L} u\left(x_{1}\right)-\mathscr{L} u^{\prime}\left(x_{1}^{\prime}\right)\right| \leq & \left(\sup _{x}\|A(x)\|+2 \varepsilon\right)\left\|u-u^{\prime}\right\| \\
& +\left|x_{1}-x_{1}^{\prime}\right| .
\end{aligned}
$$

We get that $\mathscr{L} \in \mathscr{K}_{1}$ is contraction and consequently we have the invariant manifold $r=u(x)$.

\section{Conjugacy of Noninvertible Mappings}

Definition 2. Two mappings $S, T: \mathbf{X} \rightarrow \mathbf{X}$ are conjugate, if there exists a homeomorphism $H: \mathbf{X} \rightarrow \mathbf{X}$ such that

$$
S \circ H(x)=H \circ T(x) \text {. }
$$

Definition 3. Two discrete semidynamical systems $S^{n}, T^{n}$ : $\mathbf{X} \rightarrow \mathbf{X}(n \in \mathbb{N})$ are conjugate, if there exists a homeomorphism $H: \mathbf{X} \rightarrow \mathbf{X}$ such that

$$
S^{n} \circ H(x)=H \circ T^{n}(x) .
$$

It is easily verified that two discrete semidynamical systems $S^{n}$ and $T^{n}$, generated by mappings $S$ and $T$, are conjugate if and only if the mappings $S$ and $T$ are conjugate.

Suppose that mapping (1) has an invariant manifold given by Lipschitzian mapping $u: \mathbf{E} \rightarrow$ F such that

$$
\begin{gathered}
\sup _{x}\|u(x)\| \leq \delta, \\
\left|u(x)-u\left(x^{\prime}\right)\right| \leq\left|x-x^{\prime}\right| .
\end{gathered}
$$

Our aim is to find a simpler mapping conjugated with (1). 
Theorem 4. If $\sup _{x}\left(\left\|(D g(x))^{-1}\right\|\|A(x)\|\right)+5 \varepsilon \sup _{x} \|(D g$ $(x))^{-1} \|<1$, then there exists a continuous mapping $v: \mathbf{E} \times \mathbf{B}(\delta) \rightarrow \mathbf{E}$ which is Lipschitzian with respect to the second variable such that mappings (1) and

$$
\begin{gathered}
x_{1}=X(x, u(x)), \\
r_{1}=R(x+v(x, r), r)
\end{gathered}
$$

are conjugated in a small neighbourhood of the invariant manifold $r=u(x)$.

We will seek the mapping establishing the conjugacy of (1) and (18) in the form

$$
H(x, r)=(x+v(x, r), r) .
$$

We get the following functional equation:

$$
\begin{aligned}
X(x+v(x, r), r)= & X(x, u(x)) \\
& +v(X(x, u(x)), R(x+v(x, r), r))
\end{aligned}
$$

or equivalently

$$
\begin{aligned}
v(x, r)=(D g(x))^{-1}( & D g(x) v(x, r)-X(x+v(x, r), r) \\
& +X(x, u(x)) \\
& +v(X(x, u(x)), R(x+v(x, r), r))) .
\end{aligned}
$$

The proof of the theorem consists of four lemmas.

Lemma 5. The functional equation (20) has a unique solution in $\mathscr{M}_{1}$.

Proof. The set of continuous mappings $v: \mathbf{E} \times \mathbf{B}(\delta) \rightarrow \mathbf{E}$,

$$
\mathscr{M}=\left\{v \in \mathbf{C}(\mathbf{E} \times \mathbf{B}(\delta), \mathbf{E}) \mid \sup _{x, r} \frac{|v(x, r)|}{|r-u(x)|}<+\infty\right\}
$$

becomes a Banach space if we use the norm $\|v\|=$ $\sup _{x, r}(|v(x, r)| /|r-u(x)|)$. The set

$$
\mathscr{M}_{1}=\left\{v \in \mathscr{M}|\|v\| \leq 1,| v(x, r)-v\left(x, r^{\prime}\right)|\leq| r-r^{\prime} \mid\right\}
$$

is a closed subset of the Banach space $\mathscr{M}$.

Let us consider the mapping $v \mapsto \mathscr{L} v, v \in \mathscr{M}_{1}$ defined by the equality

$$
\begin{aligned}
& \mathscr{L} v(x, r) \\
& \begin{aligned}
&=(D g(x))^{-1} v(X(x, u(x)), R(x+v(x, r), r)) \\
&+(D g(x))^{-1}(D g(x) v(x, r)-g(x+v(x, r))+g(x) \\
&-\Psi(x+v(x, r), r)+\Psi(x, u(x))) .
\end{aligned}
\end{aligned}
$$

First we obtain

$$
\begin{aligned}
&|L v(x, r)| \\
& \leq\left\|(D g(x))^{-1}\right\||R(x+v(x, r), r)-u(X(x, u(x)))| \\
&+\left\|(D g(x))^{-1}\right\||D g(x) v(x, r)-g(x+v(x, r))+g(x)| \\
&+\left\|(D g(x))^{-1}\right\||\Psi(x+v(x, r), r)-\Psi(x, u(x))| \\
& \leq\left\|(D g(x))^{-1}\right\| \\
& \times(\|A(x)\|+\gamma|r|+2 \varepsilon+\omega(|r-u(x)|)+2 \varepsilon) \\
& \times|r-u(x)| .
\end{aligned}
$$

Here we used Hadamard lemma:

$$
g\left(x^{\prime}\right)-g(x)=\int_{0}^{1} D g\left(x+\theta\left(x^{\prime}-x\right)\right) d \theta\left(x^{\prime}-x\right) .
$$

Next we get

$$
\begin{aligned}
& \left|L v(x, r)-L v\left(x, r^{\prime}\right)\right| \\
& \leq\left\|(D g(x))^{-1}\right\|\left|R(x+v(x, r), r)-R\left(x+v\left(x, r^{\prime}\right), r^{\prime}\right)\right| \\
& +\left\|(D g(x))^{-1}\right\| \mid D g(x)\left(v(x, r)-v\left(x, r^{\prime}\right)\right) \\
& \quad-g(x+v(x, r))+g\left(x+v\left(x, r^{\prime}\right)\right) \mid \\
& \quad+\left\|(D g(x))^{-1}\right\| \\
& \quad \times\left|\Psi(x+v(x, r), r)-\Psi\left(x+v\left(x, r^{\prime}\right), r^{\prime}\right)\right| \\
& \leq\left\|(D g(x))^{-1}\right\|\left(\|A(x)\|+2 \gamma \max \left\{|r-u(x)|,\left|r^{\prime}\right|\right\}+2 \varepsilon\right) \\
& \quad \times\left|r-r^{\prime}\right| \\
& \quad+\left\|(D g(x))^{-1}\right\| \\
& \quad \times\left(\omega\left(\max \left\{|r-u(x)|,\left|r^{\prime}-u(x)\right|\right\}\right)+2 \varepsilon\right)\left|r-r^{\prime}\right| .
\end{aligned}
$$

In addition,

$$
\begin{aligned}
\left|L v(x, r)-L v^{\prime}(x, r)\right| & \\
\leq \| & \left|(D g(x))^{-1} \|\right| R(x+v(x, r), r)-R\left(x+v^{\prime}(x, r), r\right) \mid \\
+\left\|(D g(x))^{-1}\right\| & \mid v(X(x, u(x)), R(x+v(x, r), r)) \\
& -v^{\prime}(X(x, u(x)), R(x+v(x, r), r)) \mid \\
+\left\|(D g(x))^{-1}\right\| & \mid D g(x)\left(v(x, r)-v^{\prime}(x, r)\right) \\
& -g(x+v(x, r))+g\left(x+v^{\prime}(x, r)\right) \mid
\end{aligned}
$$




$$
\begin{aligned}
& +\left\|(D g(x))^{-1}\right\| \\
& \times\left|\Psi(x+v(x, r), r)-\Psi\left(x+v^{\prime}(x, r), r\right)\right| \\
\leq & \left\|(D g(x))^{-1}\right\|(\|A(x)\|+2 \gamma|r|+3 \varepsilon)\left\|v-v^{\prime}\right\||r-u(x)| \\
& +\left\|(D g(x))^{-1}\right\|(\omega(|r-u(x)|)+\varepsilon) \\
& \times\left\|v-v^{\prime}\right\||r-u(x)| .
\end{aligned}
$$

We choose $\delta>0$, where $\max \left\{|r|,\left|r^{\prime}\right|\right\}=\delta \leq a$, such that

$$
\begin{aligned}
& \sup _{x}\left(\left\|(D g(x))^{-1}\right\|\|A(x)\|\right) \\
& \quad+(5 \varepsilon+\omega(8 \delta)+4 \gamma \delta) \sup _{x}\left\|(D g(x))^{-1}\right\|<1 .
\end{aligned}
$$

Then $\|\mathscr{L} v\| \leq 1,\left|\mathscr{L} v(x, r)-\mathscr{L} v\left(x, r^{\prime}\right)\right| \leq\left|r-r^{\prime}\right|$, the mapping $\mathscr{L}$ is a contraction, and consequently the functional equation (20) has unique solution in $\mathscr{M}_{1}$.

Next we will prove that the mapping $H$ is a homeomorphism in the small neighbourhood of the invariant manifold $r=u(x)$. Let us consider the functional equation

$$
\begin{aligned}
& X\left(x+v_{1}(x, r), u\left(x+v_{1}(x, r)\right)\right) \\
& \quad=X(x, r)+v_{1}(X(x, r), R(x, r))
\end{aligned}
$$

or equivalently

$$
\begin{aligned}
& v_{1}(x, r) \\
& \qquad \begin{aligned}
=(D g(x))^{-1}( & D g(x) v_{1}(x, r) \\
& -X\left(x+v_{1}(x, r), u\left(x+v_{1}(x, r)\right)\right) \\
& \left.+X(x, r)+v_{1}(X(x, r), R(x, r))\right) .
\end{aligned}
\end{aligned}
$$

Lemma 6. The functional equation (30) has a unique solution in $\mathscr{M}_{2}$.

Proof. The set

$$
\mathscr{M}_{2}=\{v \in \mathscr{M} \mid\|v\| \leq 1\}
$$

is a closed subset of the Banach space $\mathscr{M}$.

Let us consider the mapping $v_{1} \mapsto \mathscr{L} v_{1}, v_{1} \in \mathscr{M}_{2}$ defined by the equality

$$
\begin{aligned}
\mathscr{L} v_{1}(x, r) & \\
= & (D g(x))^{-1} v_{1}(X(x, r), R(x, r))+(D g(x))^{-1} \\
& \times\left(D g(x) v_{1}(x, r)-g\left(x+v_{1}(x, r)\right)+g(x)\right. \\
& \left.\quad-\Psi\left(x+v_{1}(x, r), u\left(x+v_{1}(x, r)\right)\right)+\Psi(x, r)\right) .
\end{aligned}
$$

We have

$$
\begin{aligned}
&\left|L v_{1}(x, r)\right| \\
& \leq\left\|(D g(x))^{-1}\right\||R(x, r)-u(X(x, r))|+\left\|(D g(x))^{-1}\right\| \\
& \quad \times\left|D g(x) v_{1}(x, r)-g\left(x+v_{1}(x, r)\right)+g(x)\right| \\
&+\left\|(D g(x))^{-1}\right\| \\
& \quad \times\left|\Psi\left(x+v_{1}(x, r), u\left(x+v_{1}(x, r)\right)\right)-\Psi(x, r)\right| \\
& \leq\left\|(D g(x))^{-1}\right\|(\|A(x)\|+2 \varepsilon+\omega(|r-u(x)|)+3 \varepsilon) \\
& \times|r-u(x)| .
\end{aligned}
$$

We obtain

$$
\begin{aligned}
& \left|L v_{1}(x, r)-L v_{1}^{\prime}(x, r)\right| \\
& \leq\left\|(D g(x))^{-1}\right\| \\
& \quad \times\left|v_{1}(X(x, r), R(x, r))-v_{1}^{\prime}(X(x, r), R(x, r))\right| \\
& \quad+\left\|(D g(x))^{-1}\right\| \\
& \quad \times \mid D g(x)\left(v_{1}(x, r)-v_{1}^{\prime}(x, r)\right) \\
& \quad-g\left(x+v_{1}(x, r)\right)+g\left(x+v_{1}^{\prime}(x, r)\right) \mid+\left\|(D g(x))^{-1}\right\| \\
& \quad \times \mid \Psi\left(x+v_{1}(x, r), u\left(x+v_{1}(x, r)\right)\right) \\
& \quad-\Psi\left(x+v_{1}^{\prime}(x, r), u\left(x+v_{1}^{\prime}(x, r)\right)\right) \mid \\
& \leq\left\|(D g(x))^{-1}\right\|(\|A(x)\|+2 \varepsilon+\omega(|r-u(x)|)+2 \varepsilon) \\
& \quad \times\left\|v_{1}-v_{1}^{\prime}\right\||r-u(x)| .
\end{aligned}
$$

We get that $\mathscr{L}$ is a contraction and consequently the functional equation (30) has a unique solution in $\mathscr{M}_{2}$.

Consider the mapping $G$ defined by equality $G(x, r)=$ $\left(x+v_{1}(x, r), r\right)$.

Lemma 7. One has $G \circ H=i d$.

Proof. Let us consider the functional equation

$$
\begin{aligned}
& X\left(x+v_{2}(x, r), u\left(x+v_{2}(x, r)\right)\right) \\
& \quad=X(x, u(x))+v_{2}(X(x, u(x)), R(x+v(x, r), r))
\end{aligned}
$$

or equivalently

$$
\begin{aligned}
& v_{2}(x, r) \\
& =(D g(x))^{-1} \\
& \times\left(D g(x) v_{2}(x, r)-X\left(x+v_{2}(x, r), u\left(x+v_{2}(x, r)\right)\right)\right. \\
& \left.+X(x, u(x))+v_{2}(X(x, u(x)), R(x+v(x, r), r))\right) .
\end{aligned}
$$


It is easily verified that the functional equation (36) has the trivial solution. Let us prove the uniqueness of the solution in $\mathscr{M}_{3}$, where

$$
\mathscr{M}_{3}=\left\{v_{2} \in \mathscr{M} \mid\left\|v_{2}\right\| \leq 3\right\}
$$

is a closed subset of the Banach space $\mathscr{M}$. We get

$$
\begin{aligned}
\left|v_{2}(x, r)\right| & \\
\leq & \left\|(D g(x))^{-1}\right\|\left\|v_{2}\right\||R(x+v(x, r), r)-u(X(x, u(x)))| \\
& \times \\
\quad & \times \mid D g(x))^{-1} \| \\
& +\|\left(D g(x) v_{2}(x, r)-g\left(x+v_{2}(x, r)\right)+g(x) \mid\right. \\
& \times\left|\Psi\left(x+v_{2}(x, r), u\left(x+v_{2}(x, r)\right)\right)-\Psi(x, u(x))\right| \\
\leq & \left\|(D g(x))^{-1}\right\| \\
& \times(\|A(x)\|+\gamma|r|+2 \varepsilon+\omega(3|r-u(x)|)+2 \varepsilon) \\
& \times\left\|v_{2}\right\||r-u(x)| .
\end{aligned}
$$

It follows that $v_{2}(x, r) \equiv 0$. The mapping $w_{1}$, where

$$
w_{1}(x, r)=v(x, r)+v_{1}(x+v(x, r), r),
$$

also satisfies the functional equation (36). Using the change of variables $x \mapsto x+v(x, r)$ in (30) we get

$$
\begin{aligned}
X(x & \left.+w_{1}(x, r), u\left(x+w_{1}(x, r)\right)\right) \\
= & X(x+v(x, r), r) \\
& +v_{1}(X(x+v(x, r), r), R(x+v(x, r), r)) .
\end{aligned}
$$

Using (20), we obtain

$$
\begin{gathered}
X\left(x+w_{1}(x, r), u\left(x+w_{1}(x, r)\right)\right) \\
=X(x, u(x))+v(X(x, u(x)), R(x+v(x, r), r)) \\
+v_{1}(X(x, u(x))+v(X(x, u(x)), R(x+v(x, r), r)), \\
\quad R(x+v(x, r), r)) \\
=X(x, u(x))+w_{1}(X(x, u(x)), R(x+v(x, r), r)) .
\end{gathered}
$$

Let us note that

$\left|w_{1}(x, r)\right| \leq|r-u(x)|+|r-u(x+v(x, r))| \leq 3|r-u(x)|$.

Therefore $\left\|w_{1}\right\| \leq 3$ and we have

$$
v(x, r)+v_{1}(x+v(x, r), r)=0 .
$$

We obtain that $G \circ H=$ id.
Lemma 8. One has $H \circ G=i d$.

Proof. The set of continuous mappings $v_{3}: \mathbf{E} \times \mathbf{B}(\delta) \times \mathbf{B}(\delta) \rightarrow$ E,

$$
\begin{aligned}
& \mathscr{N}=\left\{v_{3} \in \mathbf{C}(\mathbf{E} \times \mathbf{B}(\delta) \times \mathbf{B}(\delta), \mathbf{E}) \mid\right. \\
&\left.\sup _{x, r, z} \frac{\left|v_{3}(x, r, z)\right|}{\max (|r-u(x)|,|z-r|)}<\infty\right\}
\end{aligned}
$$

becomes a Banach space if we use the norm $\left\|v_{3}\right\|=$ $\sup _{x, r, z}\left(\left|v_{3}(x, r, z)\right| / \max (|r-u(x)|,|z-r|)\right)$. The set

$$
\begin{aligned}
\mathcal{N}_{1}= & \left\{v_{3} \in \mathcal{N} \mid\left\|v_{3}\right\| \leq 1,\right. \\
& \left.\left|v_{3}(x, r, z)-v_{3}\left(x, r, z^{\prime}\right)\right| \leq\left|z-z^{\prime}\right|\right\}
\end{aligned}
$$

is a closed subset of the Banach space $\mathcal{N}$.

Let us consider the functional equation

$$
\begin{aligned}
& X(x, r)+v_{3}\left(X(x, r), R(x, r), R\left(x+v_{3}(x, r, z), z\right)\right) \\
& \quad=X\left(x+v_{3}(x, r, z), z\right)
\end{aligned}
$$

or equivalently

$$
\begin{aligned}
v_{3}(x, r, z) & \\
= & (D g(x))^{-1} \\
\quad & \times\left(D g(x) v_{3}(x, r, z)-g\left(x+v_{3}(x, r, z)\right)+g(x)\right. \\
& \quad+\Psi(x, r)-\Psi\left(x+v_{3}(x, r, z), z\right) \\
& \left.\quad+v_{3}\left(X(x, r), R(x, r), R\left(x+v_{3}(x, r, z), z\right)\right)\right) .
\end{aligned}
$$

Let us consider the mapping $v_{3} \mapsto \mathscr{L} v_{3}, v_{3} \in \mathscr{N}_{1}$ defined by the equality

$$
\begin{aligned}
\mathscr{L} v_{3}(x, r, z) \\
=(D g(x))^{-1} \\
\quad \times\left(D g(x) v_{3}(x, r, z)-g\left(x+v_{3}(x, r, z)\right)+g(x)\right. \\
\quad-\Psi\left(x+v_{3}(x, r, z), z\right)+\Psi(x, r) \\
\left.\quad+v_{3}\left(X(x, r), R(x, r), R\left(x+v_{3}(x, r, z), z\right)\right)\right) .
\end{aligned}
$$

We obtain

$$
\begin{aligned}
\left|L v_{3}(x, r, z)\right| & \\
\leq\left\|(D g(x))^{-1}\right\| \max \{ & |R(x, r)-u(X(x, r))|, \\
& \left.\left|R\left(x+v_{3}(x, r, z), z\right)-R(x, r)\right|\right\}
\end{aligned}
$$




$$
\begin{aligned}
& +\left\|(D g(x))^{-1}\right\| \\
& \times\left|D g(x) v_{3}(x, r, z)-g\left(x+v_{3}(x, r, z)\right)+g(x)\right| \\
& +\left\|(D g(x))^{-1}\right\|\left|\Psi(x, r)-\Psi\left(x+v_{3}(x, r, z), z\right)\right| \\
& \leq\left\|(D g(x))^{-1}\right\|(\|A(x)\|+\gamma|z|+2 \varepsilon) \\
& \quad \times \max \{|r-u(x)|,|z-r|\}+\left\|(D g(x))^{-1}\right\| \\
& \quad \times(\omega(\max \{|r-u(x)|,|z-r|\})+2 \varepsilon) \\
& \quad \times \max \{|r-u(x)|,|z-r|\} .
\end{aligned}
$$

In addition,

$$
\begin{aligned}
\mid L v_{3}( & x, r, z)-L v_{3}\left(x, r, z^{\prime}\right) \mid \\
\leq & \left\|(D g(x))^{-1}\right\| \\
& \times \mid D g(x)\left(v_{3}(x, r, z)-v_{3}\left(x, r, z^{\prime}\right)\right) \\
& -g\left(x+v_{3}(x, r, z)\right)+g\left(x+v_{3}\left(x, r, z^{\prime}\right)\right) \mid \\
& +\left\|(D g(x))^{-1}\right\| \\
& \times\left|\Psi\left(x+v_{3}(x, r, z), z\right)-\Psi\left(x+v_{3}\left(x, r, z^{\prime}\right), z^{\prime}\right)\right| \\
& +\left\|(D g(x))^{-1}\right\| \\
& \times\left|R\left(x+v_{3}(x, r, z), z\right)-R\left(x+v_{3}\left(x, r, z^{\prime}\right), z^{\prime}\right)\right| \\
\leq & \left\|(D g(x))^{-1}\right\| \\
& \times\left(\omega\left(\max \left\{|r-u(x)|,|z-r|,\left|z^{\prime}-r\right|\right\}\right)+2 \varepsilon\right)\left|z-z^{\prime}\right| \\
& +\left\|(D g(x))^{-1}\right\| \\
& \times\left(\|A\|+2 \varepsilon+2 \gamma \max \left\{|r-u(x)|,|z|,\left|z^{\prime}-r\right|\right\}\right) \\
\times & \left|z-z^{\prime}\right| \cdot
\end{aligned}
$$

Let $v_{3} \in \mathcal{N}_{1}$ and $v_{3}^{\prime} \in \mathcal{N}_{1} \cup \mathcal{N}_{2}$ where

$$
\begin{aligned}
\mathcal{N}_{2}= & \left\{v_{3}^{\prime} \in \mathcal{N}\left|\sup _{x,|r| \leq \delta,|z| \leq \delta}\right| v_{3}^{\prime}(x, r, z) \mid \leq 8 \delta,\right. \\
& \left.\left|v_{3}^{\prime}(x, r, z)-v_{3}^{\prime}\left(x, r, z^{\prime}\right)\right| \leq\left|z-z^{\prime}\right|\right\} .
\end{aligned}
$$

We have

$$
\begin{aligned}
\left|L v_{3}(x, r, z)-L v_{3}^{\prime}(x, r, z)\right| \\
\leq \| \\
\quad \times \mid D g(x))^{-1} \| \\
\quad-\operatorname{Dg}(x)\left(v_{3}(x, r, z)-v_{3}^{\prime}(x, r, z)\right) \\
\quad-g\left(x+v_{3}(x, r, z)\right)+g\left(x+v_{3}^{\prime}(x, r, z)\right) \mid
\end{aligned}
$$

$$
\begin{aligned}
& +\left\|(\operatorname{Dg}(x))^{-1}\right\| \\
& \times\left|\Psi\left(x+v_{3}(x, r, z), z\right)-\Psi\left(x+v_{3}^{\prime}(x, r, z), z\right)\right| \\
& +\left\|(\operatorname{Dg}(x))^{-1}\right\| \\
& \times \mid v_{3}\left(X(x, r), R(x, r), R\left(x+v_{3}(x, r, z), z\right)\right) \\
& -v_{3}^{\prime}\left(X(x, r), R(x, r), R\left(x+v_{3}(x, r, z), z\right)\right) \\
& +\left\|(\operatorname{Dg}(x))^{-1}\right\| \\
& \times \mid v_{3}^{\prime}\left(X(x, r), R(x, r), R\left(x+v_{3}(x, r, z), z\right)\right) \\
& -v_{3}^{\prime}\left(X(x, r), R(x, r), R\left(x+v_{3}^{\prime}(x, r, z), z\right)\right) \mid \\
& \leq\left\|(D g(x))^{-1}\right\|(\omega(\max \{|r-u(x)|,|z-r|, 8 \delta\})+\varepsilon) \\
& \times\left\|v_{3}-v_{3}^{\prime}\right\| \max \{|r-u(x)|,|z-r|\}+\left\|(D g(x))^{-1}\right\| \\
& \times \max \{|R(x, r)-u(X(x, r))|, \\
& \left.\left|R\left(x+v_{3}^{\prime}(x, r, z), z\right)-R(x, r)\right|\right\}\left\|v_{3}-v_{3}^{\prime}\right\| \\
& +\left\|(\operatorname{Dg}(x))^{-1}\right\| \\
& \times\left|R\left(x+v_{3}(x, r, z), z\right)-R\left(x+v_{3}^{\prime}(x, r, z), z\right)\right| \\
& \leq\left\|(D g(x))^{-1}\right\|(\omega(8 \delta)+\varepsilon)\left\|v_{3}-v_{3}^{\prime}\right\| \\
& \times \max \{|r-u(x)|,|z-r|\} \\
& +\left\|(D g(x))^{-1}\right\|(\|A\|+3 \varepsilon+\gamma|z|) \\
& \times \max \{|r-u(x)|,|z-r|\}\left\|v_{3}-v_{3}^{\prime}\right\| \\
& =\left\|(D g(x))^{-1}\right\|(\|A(x)\|+4 \varepsilon+\omega(8 \delta)+\gamma|z|) \\
& \times\left\|v_{3}-v_{3}^{\prime}\right\| \max \{|r-u(x)|,|z-r|\} .
\end{aligned}
$$

Then $\left\|\mathscr{L} v_{3}\right\| \leq 1,\left|\mathscr{L} v_{3}(x, r, z)-\mathscr{L} v_{3}\left(x, r, z^{\prime}\right)\right| \leq\left|z-z^{\prime}\right|$, the mapping $\mathscr{L}$ is a contraction, and consequently the functional equation (47) has a unique solution in $\mathcal{N}_{1}$. Moreover, this solution is also unique in the closed subset $\mathcal{N}_{2}$. Let us note that

$$
v_{3}(x, r, r)=0 .
$$

The mapping $w_{2}$, where

$$
w_{2}(x, r, z)=v_{1}(x, r)+v\left(x+v_{1}(x, r), z\right),
$$

satisfies (47). Using the change of variables $(x, r) \mapsto(x+$ $\left.v_{1}(x, r), z\right)$ in $(20)$ we get

$$
\begin{aligned}
X(x & \left.+w_{2}(x, r, z), z\right) \\
= & X\left(x+v_{1}(x, r), 0\right) \\
& +v\left(X\left(x+v_{1}(x, r), 0\right), R\left(x+w_{2}(x, r, z), z\right)\right) .
\end{aligned}
$$


Using (30) we obtain

$$
\begin{aligned}
X(x+ & \left.w_{2}(x, r, z), z\right) \\
= & X(x, r)+v_{1}(X(x, r), R(x, r)) \\
& +v\left(X(x, r)+v_{1}(X(x, r), R(x, r)),\right. \\
& \left.R\left(x+w_{2}(x, r, z), z\right)\right) \\
= & X(x, r) \\
& +w_{2}\left(X(x, r), R(x, r), R\left(x+w_{2}(x, r, z), z\right)\right) .
\end{aligned}
$$

Let us note that

$$
\begin{aligned}
\mid w_{2}(x, r, z) & -w_{2}\left(x, r, z^{\prime}\right)|\leq| z-z^{\prime} \mid, \\
\left|w_{2}(x, r, z)\right| \leq & |r-u(x)|+|z-r| \\
& +\left|r-u\left(x+v_{1}(x, r)\right)\right| \\
\leq & 4 \max \{|r-u(x)|,|z-r|\} .
\end{aligned}
$$

Therefore $w_{2} \in \mathcal{N}_{2}$ and we have

$$
v_{1}(x, r)+v\left(x+v_{1}(x, r), r\right)=0 .
$$

It follows that $H \circ G=\mathrm{id}$.

Finally we conclude that the mapping $H$ is a homeomorphism establishing a conjugacy of the noninvertible mappings (1) and (18).

\section{Conflict of Interests}

The author declares that there is no conflict of interests regarding the publication of this paper.

\section{Acknowledgment}

This work was partially supported by Grant no. 345/2012 of the Latvian Council of Science.

\section{References}

[1] B. Aulbach and B. M. Garay, "Linearization and decoupling of dynamical and semidynamical systems," in The Second Colloquium on Differential Equations, D. Bainov and V. Covachov, Eds., pp. 15-27, World Scientific, Singapore, 1992.

[2] B. Aulbach and B. M. Garay, "Linearizing the expanding part of noninvertible mappings," Zeitschrift für Angewandte Mathematik und Physik, vol. 44, no. 3, pp. 469-494, 1993.

[3] B. Aulbach and B. M. Garay, "Partial linearization for noninvertible mappings," Zeitschrift für Angewandte Mathematik und Physik, vol. 45, no. 4, pp. 505-542, 1994.

[4] A. Reinfelds, "Partial decoupling for noninvertible mappings," Differential Equations and Dynamical Systems, vol. 2, no. 3, pp. 205-215, 1994.

[5] A. Reinfelds, "The reduction principle for discrete dynamical and semidynamical systems in metric spaces," Zeitschrift für Angewandte Mathematik und Physik, vol. 45, no. 6, pp. 933-955, 1994.
[6] A. Reinfelds, "Partial decoupling of semidynamical system," in Mathematics, vol. 593, pp. 54-61, Latvia University, Riga, Latvia, 1994.

[7] A. Reinfelds, "The reduction of discrete dynamical and semidynamical systems in metric spaces," in Six Lectures on Dynamical Systems, B. Aulbach and F. Colonius, Eds., pp. 267-312, World Scientiffc, Singapore, 1996.

[8] A. Reinfelds, "Partial decoupling of semidynamical system in metric space," Journal of the Technical University at Plovdiv, vol. 5, pp. 33-40, 1997.

[9] A. Reinfelds, "Conjugacy of discrete semidynamical systems in the neighbourhood of invariant manifold," in Differential and Difference Equations With Applications, S. Pinelas, M. Chipot, and Z. Dosla, Eds., vol. 47 of Springer Proceedings in Mathematics and Statistics, pp. 571-578, Springer, New York, NY, USA, 2013.

[10] Ju. I. Neimark, "Existence and structural stability of invariant manifolds of pointwise mappings," Izvestiya Vysshikh Uchebnykh Zavedeniŭ, vol. 10, pp. 311-320, 1967 (Russian).

[11] R. J. Sacker, On Invariant Surface and Bifurcation of Periodic Solution of Ordinary Differential Equations, vol. 333, New York University, 1964. 


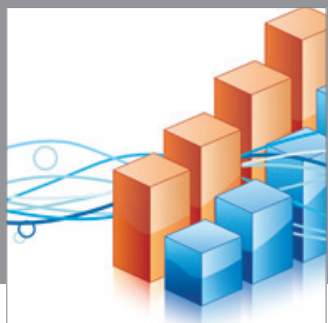

Advances in

Operations Research

mansans

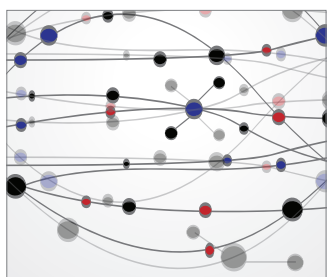

The Scientific World Journal
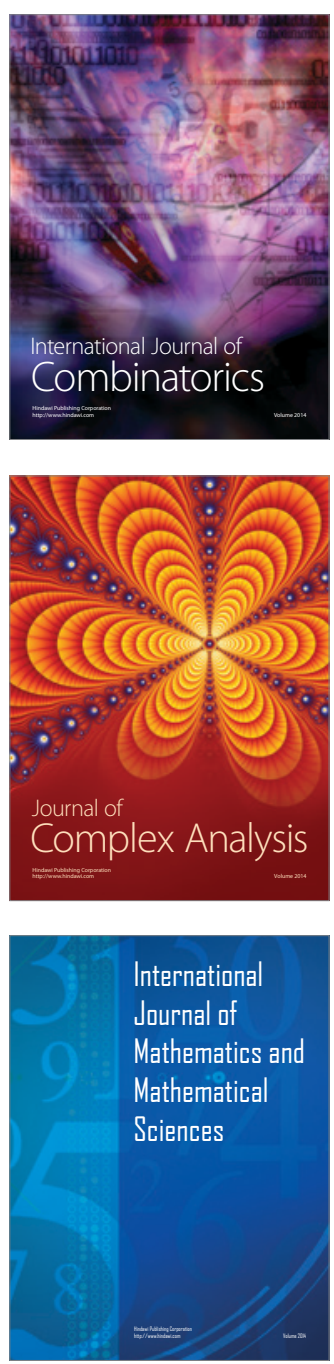
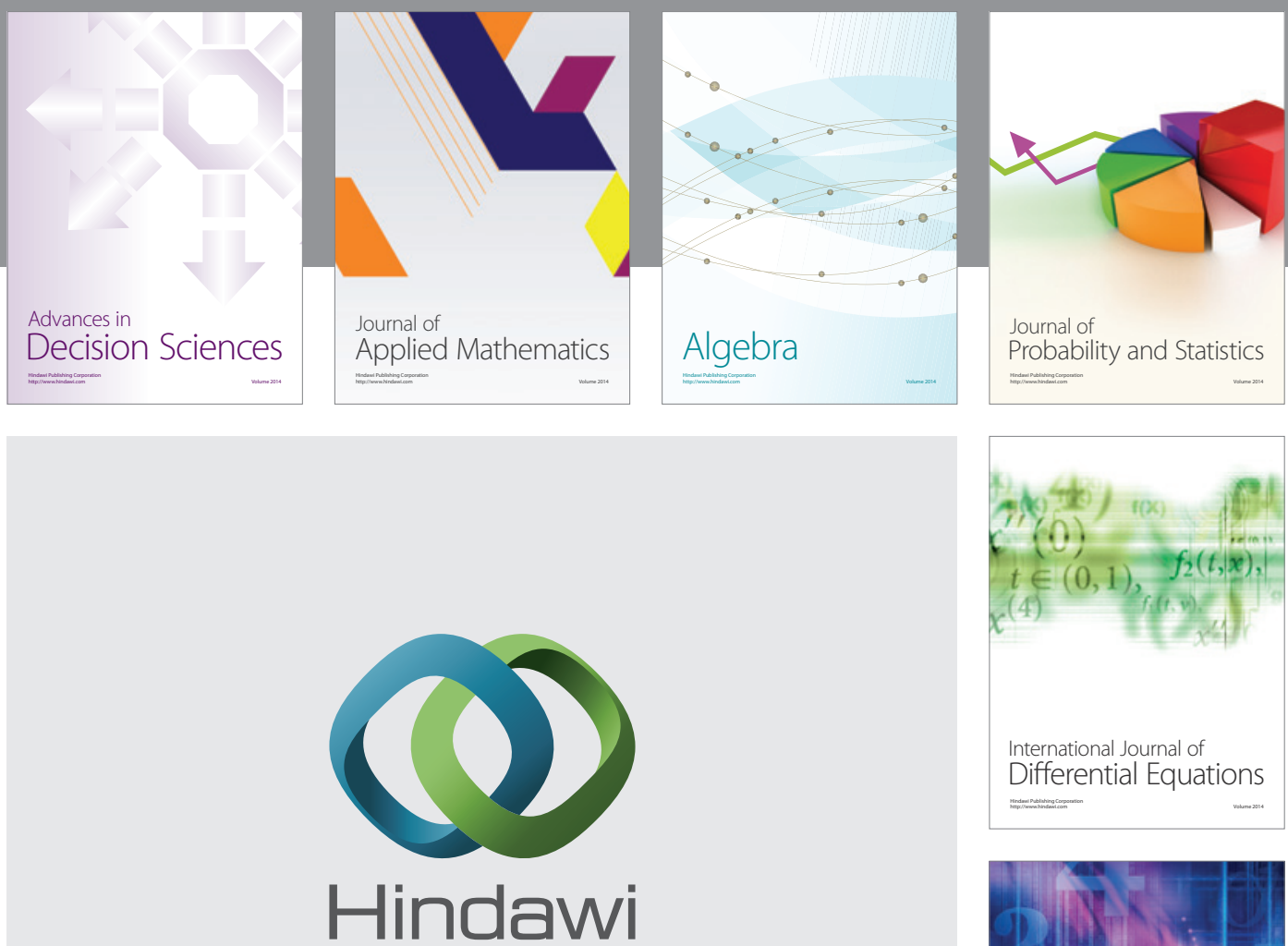

Submit your manuscripts at http://www.hindawi.com
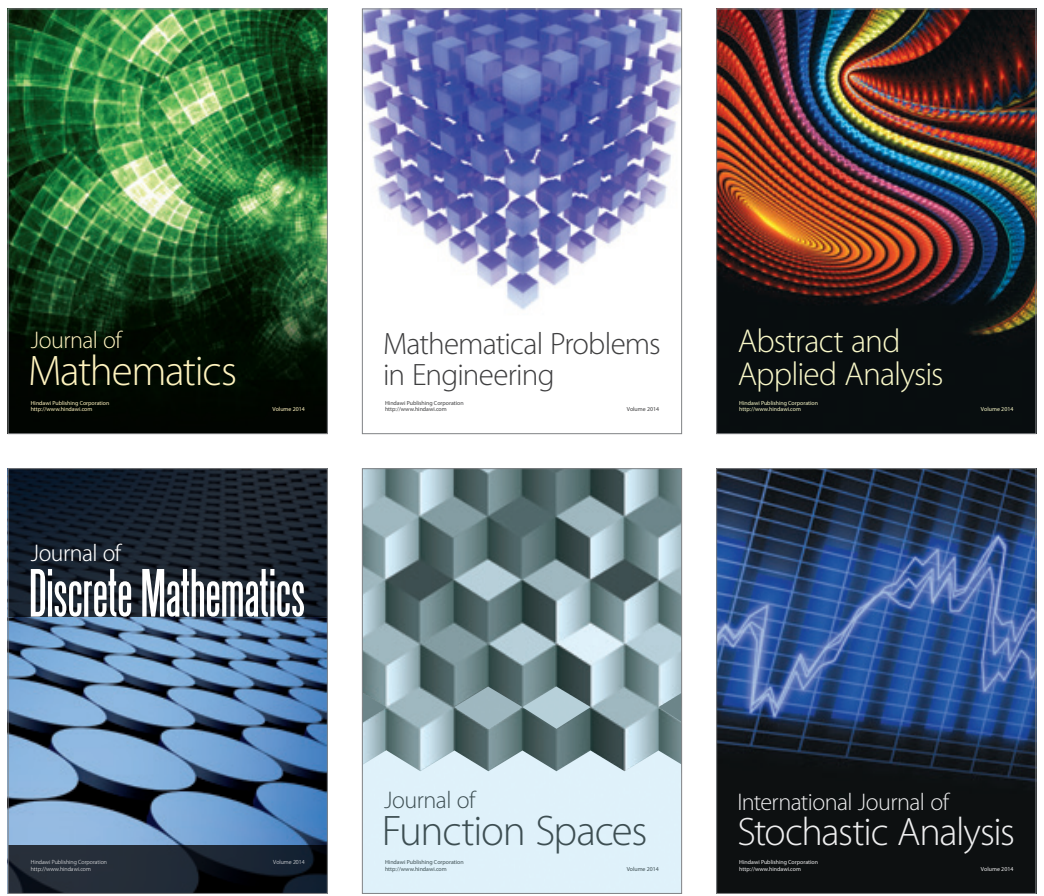

Journal of

Function Spaces

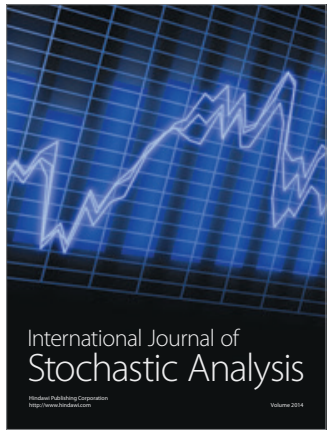

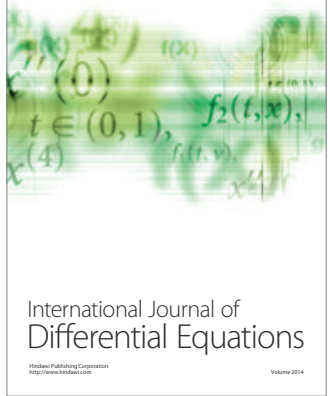
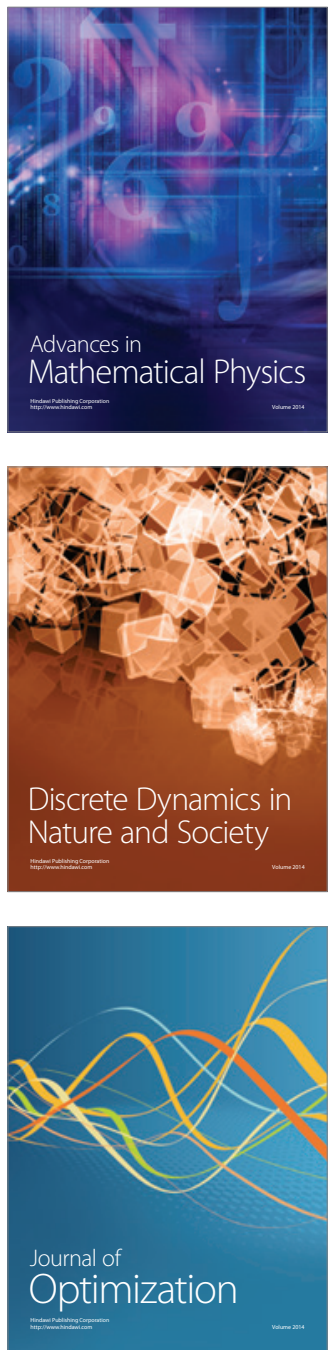